\title{
Microstructure correlation in high-strength steels with continuous stiffness mode nanoindentation results. High-resolution vs. low-resolution nanoindentation
}

\begin{abstract}
This study utilizes instrumented nanoindentation to compare the mechanical response of a transformation-induced plasticity (TRIP) steel to that of a quench and partition steel $(\mathrm{QP})$. The nanoindenter was operated using continuous stiffness mode, and the microstructure under the indent revealed by chemical etching was examined. Examination of the microstructure in the indents by scanning electron microscopy allows a direct correlation with nanoindentation properties. It is found that the hardness measured at an indentation depth of $1.1 \mu \mathrm{m}$ is, on average, significantly greater in the QP steel than in the TRIP steel. The continuous hardness measurement also allows the microstructure observed at the center of the indent to be correlated with the hardness values when indentation depths are $<0.1 \mu \mathrm{m}$. This high-resolution hardness measurement allows for a direct comparison of mechanical response for the individual retained austenite phase particles in the TRIP steel with the strengthening microconstituent in the QP steel. With this methodology, the surprising result is that the TRIP retained austenite particles have a higher hardness than the QP hardening microconstituent. It is proposed that the resolution of the apparent disagreement between the hardness measurements obtained at $1.1 \mu \mathrm{m}$ depth (QP has the higher hardness) and the 0.1- $\mu \mathrm{m}$ depth hardness measurements of the microconstituents containing retained austenite (TRIP has the higher hardness) lies in the volume fraction of the microconstituent as the QP has a two- to threefold higher volume fraction of hardening phase as compared to TRIP.
\end{abstract}

Keywords: nanoindentation; quench and partition steel; transformation-induced plasticity.

\footnotetext{
*Corresponding author: Stephen A. Hackney, Department of Materials Science and Engineering, Michigan Technological University, Houghton, MI, USA, e-mail: hackney@mtu.edu John R. Bradley (retired): General Motors, Michigan Technological University, Clarkston, MI, USA

Thomas D. Wood: Department of Materials Science and Engineering, Michigan Technological University, Houghton, MI, USA Ibrahim Miskioglu: Department of Mechanical Engineering and Engineering Mechanics, Michigan Technological University, Houghton, MI, USA
}

\section{Introduction}

The development of the continuous stiffness mode of operation $[1,2]$ for instrumented nanoindentation allows the hardness to be measured continuously as a function of depth of indentation. This type of continuous hardness measurement has already been used to study the hardness variation with indentation depth for the case of layered films, where the mechanical properties vary from layer to layer [2]. We now extend this concept to examine the implication of multi-phase distributions on continuous hardness results. Consider a Berkovich indenter tip used to indent a material with complex, spatially varying microstructure. At the point of contact between the sample surface and the tip, the measured hardness will be a function of the rounded tip of the trigonal pyramid, the surface roughness and the microstructure/materials properties under that tip [3, 4]. As the pyramid pushes into the material, the strain field becomes self-similar having volume directly correlated with the depth of indentation with maximum strain under the center of the indent [5]. If the microstructure characteristic length scale is similar to or larger than the length scale of the strain field at maximum indentation depth, then we should expect the hardness to be a function of the indentation depth due to microstructure variation in addition to the well-known plastic field geometry effects. That is, the measured hardness becomes a function of the microstructure distribution within the strain field, with the microstructure at the center of the indent being the most heavily weighted. This suggests that, if the microstructure under the center of the indent can be revealed and correlated to the hardness at low indentation depth, then a powerful tool for high-resolution hardness measurement of materials microstructure can be utilized. In this paper, such a methodology is applied to compare the 'low-resolution' hardness and 'high-resolution' hardness of a quench and partition (QP) steel [6] with those properties of a transformation-induced plasticity (TRIP) steel. 

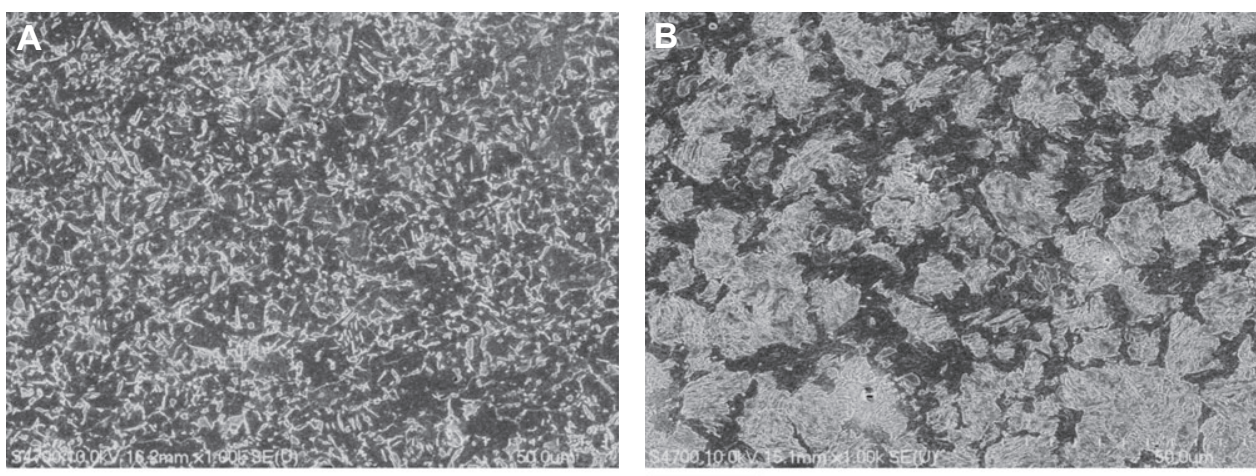

\section{$50 \mu \mathrm{m}$}

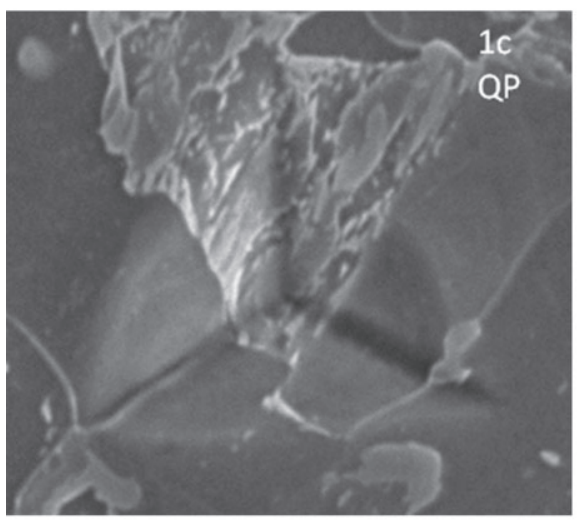

\section{$50 \mu \mathrm{m}$}
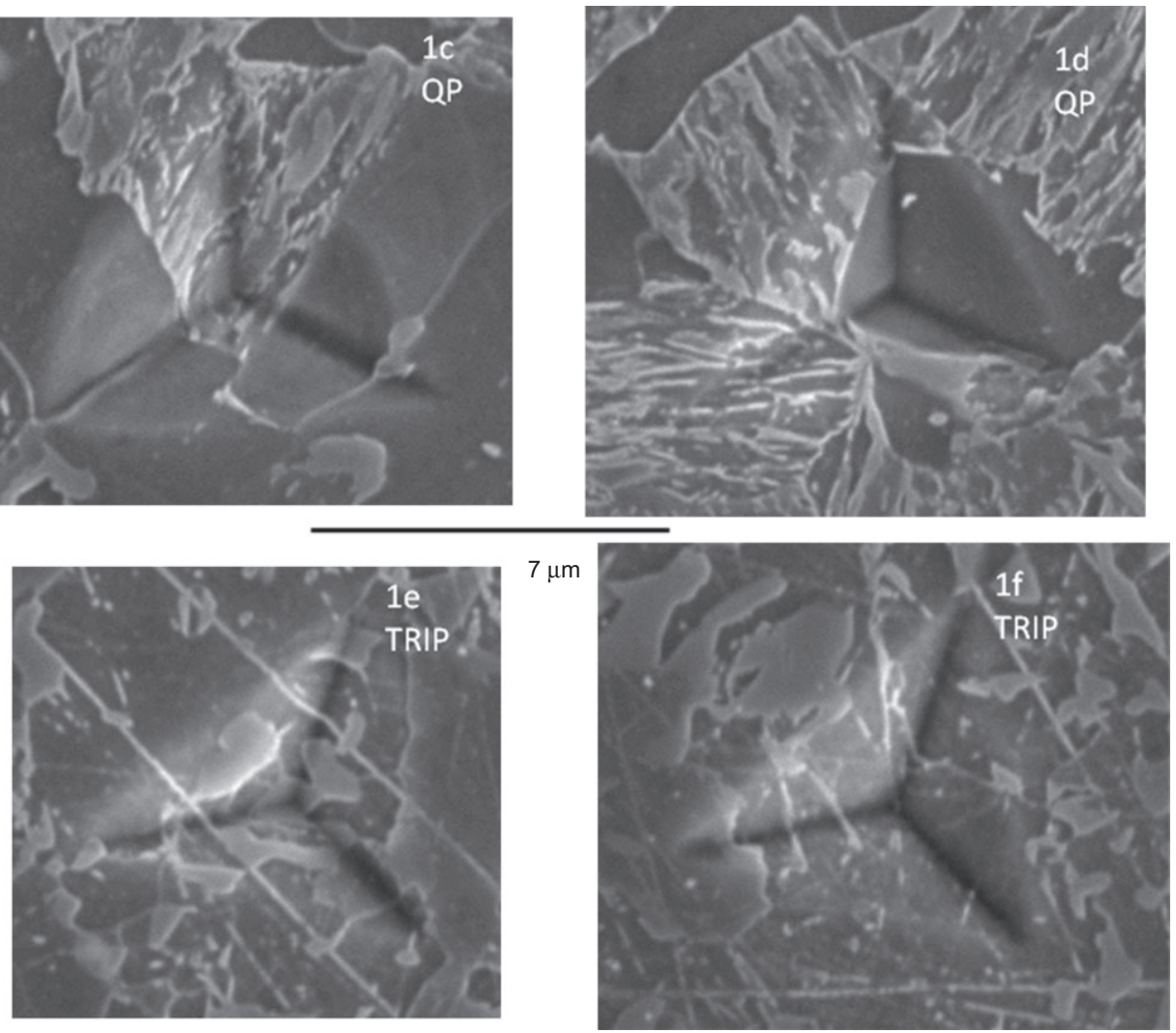

Figure 1 (A) TRIP steel etch microstructure. The retained autenite is seen as the lighter component with area fraction of 0.3. (B) QP steel etch microstructure. The lighter component contains retained austenite. The area fraction of the lighter component is 0.64 . (C) Indent microstructure showing strengthening phase at the center of the indent. (D) Indent microstructure showing ferrite at the center of the indent. (E) Indent microstructure showing strengthening phase (retained austenite) at the center of the indent. (F) Indent microstructure showing ferrite at the center of the indent.

\section{Experimental procedure}

Mounted samples of advanced high-strength QP and TRIP steels were mechanically polished with $0.05 \mu \mathrm{m} \mathrm{Al}_{2} \mathrm{O}_{3}$ as the final step, followed by a heavy etch in $2 \%$ nital, followed by repolishing with $0.05 \mu \mathrm{m} \mathrm{Al}_{2} \mathrm{O}_{3}$ to remove the etch layer and produce a relatively smooth surface. A $5 \times 5$ grid of Berkovich tip indents to a depth of $1.1 \mu \mathrm{m}$ was placed on each sample, with indent spacing of $50 \mu \mathrm{m}$ along a row of indents and $100 \mu \mathrm{m}$ of row spacing in orthogonal directions. The indentation process was followed by a $2 \%$ nital etch to reveal the microstructure. The residual indent area and area fraction of hardening microconstituent within the indent are measured from the scanning electron microscopy images. It is also noted whether the center of the indent is composed of a ferrite grain or a hardening microconstituent. This allows the microstructure observed at the center of the indent to be 
A
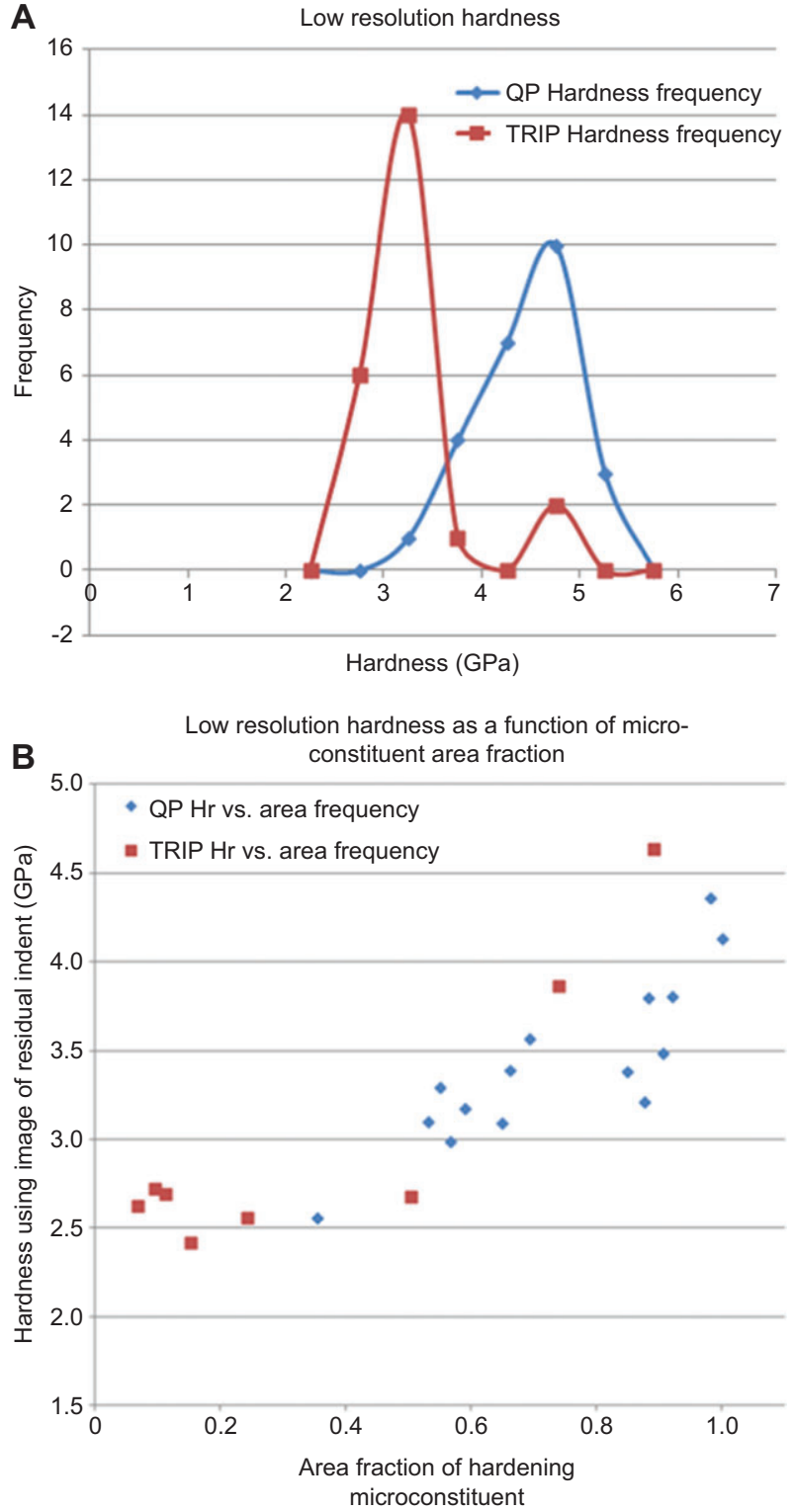

Figure 2 (A) Frequency of observation for average hardness values as determined over the entire depth of indentation, with maximum indentation depth at $1.1 \mu \mathrm{m}$. (B) Low-resolution hardness for TRIP and $Q P$ as a function of area fraction of microconstituent that is not ferrite grain.

correlated with hardness measured at very small indentation depths using the continuous stiffness measurement mode of operation.

\section{Results}

In the continuous stiffness mode of operation, the nanoindenter gives a measurement for hardness at each step of the indentation depth. In this work, the hardness at indentation depths of $<0.1 \mu \mathrm{m}$ (termed high-resolution hardness) and that at indentation depths of $1.1 \mu \mathrm{m}$ (termed low-resolution hardness) are of interest. The high- and low-resolution indentation hardness are correlated with the microstructure within the indent. The microstructure at the center of the indent makes the major contribution to the behavior of the hardness in the initial stages of the indentation process, when the indentation depth (and thus indentation area) is relatively small, and will be correlated with the high-resolution indentation hardness. The microstructure within the entire indent contributes to the hardness observed at the end of the indentation process (low-resolution hardness) when the indent depth approaches $1.1 \mu \mathrm{m}$.

The microstructures of the steels have been studied by many previous investigations but are reexamined here to aid the discussion. The microstructures are revealed by etching as shown in Figure 1. The lighter areas (raised areas appear lighter) are interpreted as retained austenite in TRIP steels. The lighter areas in the QP steel are interpreted as either martensite, bainite or retained austenite. The retained austenite is thought to exist within the martensite and bainite structures, in addition to individual austenite particles. Thus the lighter regions in the etched microstructures of both steels contain the strengthening phases, with the austenite phase contributing to strengthening through martensite transformation under applied strain. The darker regions in both steels are interpreted as ferrite, which is a softer, more ductile phase.

As can be seen in Figure 2A, the hardness values for the low-resolution hardness indicate that the QP steel is generally harder than the TRIP steel. A correlation between the variations in hardness in Figure 2A with area fraction of hardening microconstituent within the indent is shown in Figure 2B. In general, an increase in hardness with area fraction of hardening phase is indeed observed. The statistics for a straight line fit of the data is shown in Table 1. Comparison between TRIP and QP is somewhat hampered because the area fraction of hardening microconstituent in the TRIP (0.3) is significantly < for the QP (0.64), as confirmed by lowmagnification image analysis of etched samples.

The high-resolution hardness is based on the continuous hardness measurements examined for indentation depth of $<0.1 \mu \mathrm{m}$. The hardness behavior at this small indentation depth is correlated to the microstructure at the

Table 1 Statistics of best line fit for Figure 2B data.

\begin{tabular}{llr}
\hline Line fit & TRIP & QP \\
\hline Slope (GPa) & 2.19 & 2.01 \\
Intercept (GPa) & 2.25 & 1.95 \\
Correlation & 0.89 & 0.85 \\
\hline
\end{tabular}




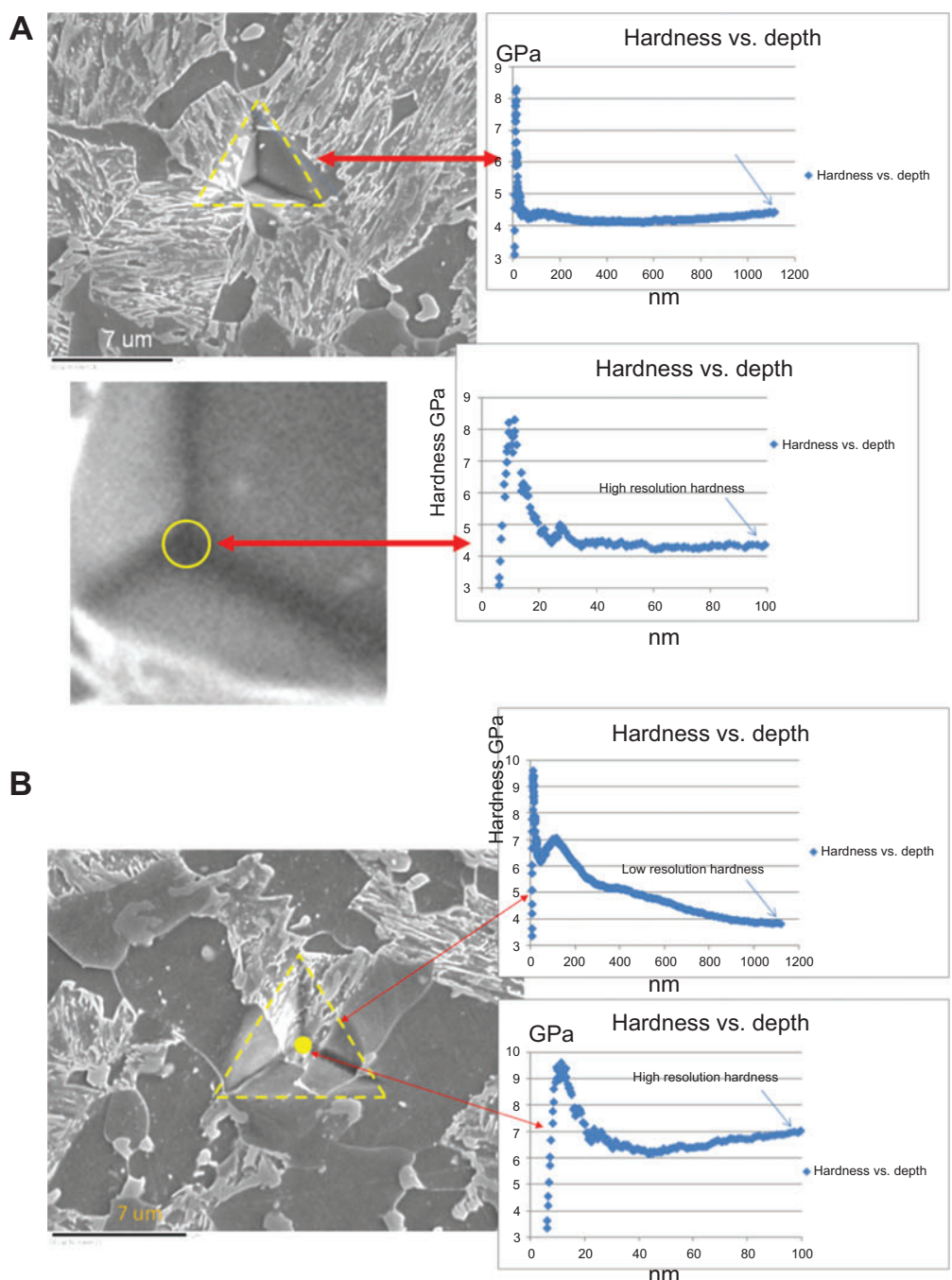

C

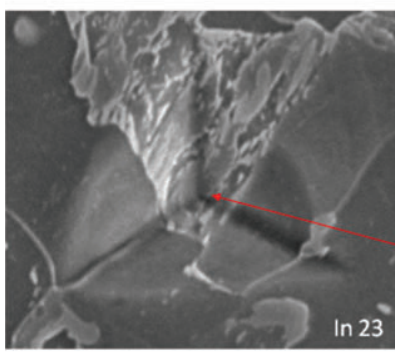

Comparision of QP high resolution hardness as a function of microstructure
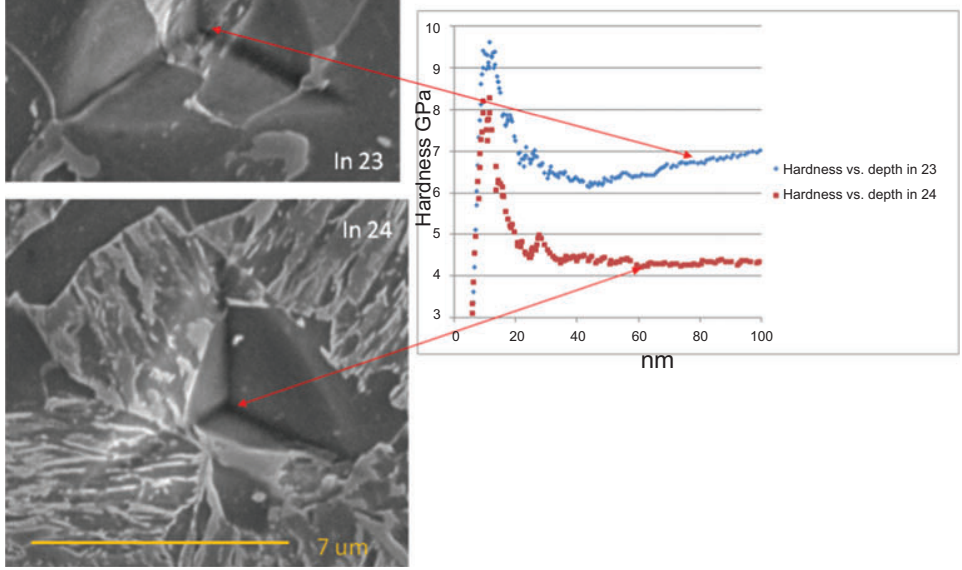

Figure 3 (A) Example of QP continuous hardness measurement increasing as the area fraction of strengthening phase under the indent increases as a function of indentation depth. (B) Example of QP continuous hardness measurement decreasing as the area fraction of strengthening phase under the indent decreases as a function of indentation depth. (C) Comparison of the QP high-resolution indentation continuous hardness measurement for the soft ferrite and relatively hard martensite/retained austenite microconstituents at the center of the indents. 

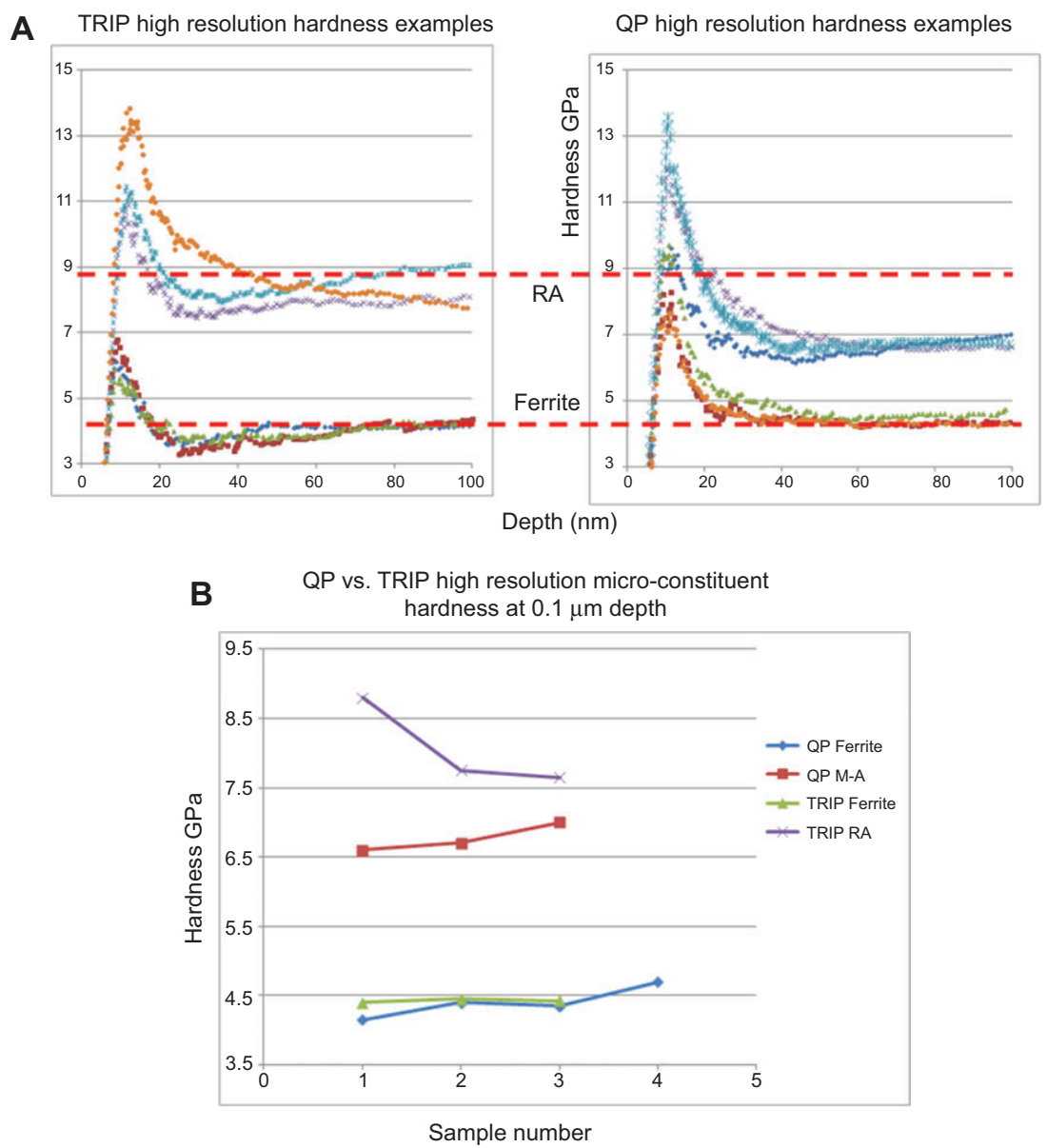

Figure 4 (A) High-resolution continuous hardness measurement for QP vs. TRIP steels. Although the high-resolution ferrite hardness indicated by the lower red dotted line is similar between QP and TRIP, the hardening microconstituent (RA) in QP has a comparatively lower hardness. (B) High-resolution hardness of the various microconstituents including ferrite grains, TRIP residual austenite (RA) and QP martensite-retained austenite (M-A).

center of the indent. The high-resolution hardness behavior can be separated based on whether the center of the indent is a ferrite grain or a hardening microconstituent (Figures 3 and 4). This is demonstrated for the QP steel in Figure 3, where the hardness at low penetration depth is seen to be a function of the microstructure at the center of the indent, whereas the hardness at higher penetration depths is seen to be a function of the microstructure of the entire area under the indent. The results in Figures 3 and 4 reinforce what was presented in Figure 2, namely, that the hardness increases as a function of the area of the hardening microconstituent under the indent. When comparing the high-resolution hardness results of the QP and TRIP steels, there is a reproducible measurement for ferrite hardness between the two samples, but a significant difference in the retained austenite microconstituent hardness (Figure 4A). The continuous hardness data from Figure 4A is reduced to that in Figure 4B by averaging the hardness measured from 90 to $100 \mathrm{~nm}$ of indentation depth. This avoids influence from the noisy set of data for indentation up to $25 \mathrm{~nm}$, where the influence of surface roughness and the elastic/plastic transition is thought to be predominant. As shown in Figure 4B, the ferrite high-resolution hardness acts as an internal standard for the validation of the highresolution hardness measurements. Using the ferrite highresolution hardness as a baseline, Figure 4B shows that the hardening microconstituent in TRIP has a significantly higher hardness than that found in the QP steel. This is in contrast to the low-resolution hardness shown in Figure 2. A rationalization for this apparent paradox arising from comparison of Figure 2A and Figure $4 \mathrm{~B}$ is that there is a low volume fraction of hardening microconstituent in TRIP as compared to the QP steel (Table 1).

Comparing Figures 2, 3 and 4, it is also observed that the high-resolution hardness values are much greater than the low-resolution hardness values for both QP and TRIP steels. This could be expected from the well-known indentation size effect $[7,8]$. 


\section{Conclusion}

This paper demonstrates that continuous stiffness mode operation of instrumented nanoindentation can allow for high-resolution hardness measurements of nonuniform microstructures at $0.1 \mu \mathrm{m}$ indentation depth. Significant differences are observed for the hardness behavior of the retained austenite microconstituent in QP vs. TRIP steels as measured using submicron spatial resolution correlations of the microstructure/hardness relationship.

\section{References}

[1] Oliver WC, Pharr GM. J. Mater. Res. 1992, 7, 1564-1583.

[2] Li X, Bhushan B. Mater. Character. 2002, 48, 11-36.

[3] Taylor CR, Malshe AP, Salamo G, Prince RN, Riester L, Cho SO. Smart Mater. Struct. 2005, 14, 963-970.

[4] Kim JY, Lee BW, Read DT, Kwon D. Scripta Mater. 2005, 52, 353-358.

[5] Larsson PL. Philos. Mag. 2006, 86, 33-35.

[6] Speer JG, Streicher AM, Matlock DK, Rizzo FC, Krauss G. In: Austenite Formation and Decomposition, Damm EB, Merwin M, Eds., TMS/ISS: Warrendale, PA, 2003, pp. 505-522.

[7] Pethica JB, Hutchings R, Oliver WC. Philos. Mag. A. 1983, 48, 593-599.

[8] Doerner MF, Nix WD. J. Mater. Res. 1986, 1, 601-608. 